Canadian

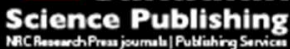

Canadian Journal of Zoology Revue canadienne de zoologie

\title{
Hybridization of domestic mink with wild American mink (Neovison vison) in eastern Canada
}

\begin{tabular}{|r|l|}
\hline Journal: & Canadian Journal of Zoology \\
\hline Manuscript ID & cjz-2016-0171.R1 \\
\hline Danuscript Type: & Article \\
\hline Complete List of Authors: & $\begin{array}{l}\text { Bowman, Jeff; Ontario Ministry of Natural Resources, Wildlife Research and } \\
\text { Development Section } \\
\text { Beauclerc, Kaela; Ontario Ministry of Natural Resources and Forestry } \\
\text { Farid, A.; Dalhousie University Faculty of Agriculture } \\
\text { Fenton, Heather; University of Georgia } \\
\text { Klutsch, Cornelya; Trent University, Biology } \\
\text { Schulte-Hostedde, Albrecht; Laurentian University, Biology }\end{array}$ \\
\hline Keyword: & $\begin{array}{l}\text { Domestication, Hybridization, Introgression, Neovison vison, insular, } \\
\text { American mink }\end{array}$ \\
\hline & \\
\hline
\end{tabular}


Hybridization of domestic mink with wild American mink (Neovison vison) in eastern Canada. Jeff Bowman, Kaela Beauclerc, A. Hossain Farid, Heather Fenton, Cornelya F. C. Klütsch, and

3 Albrecht I. Schulte-Hostedde

4

5 Jeff Bowman ${ }^{1}$ and Kaela Beauclerc. Wildlife Research and Monitoring Section, Trent University DNA Building, Peterborough, ON, K9L OG2

A. Hossain Farid. Department of Plant \& Animal Sciences, Dalhousie University, Faculty of Agriculture, PO Box 550, Truro, NS, B2N 5 E3

Heather Fenton*. Canadian Wildlife Health Cooperative, Atlantic Veterinary College, University of Prince Edward Island, 550 University Avenue, Charlottetown, PEI, C1A 4P3

Cornelya F. C. Klütsch. Biology Department, Trent University, Peterborough, Ontario, K9L 0G2

Albrecht I. Schulte-Hostedde. Department of Biology Laurentian University, Ramsey Lake Rd.,

13 Sudbury, ON, P3E 2C6

$14 \quad{ }^{1}$ Corresponding author (email: jeff.bowman@ontario.ca).

15 *Present address. 589 D. W. Brooks Drive, Wildlife Health Building, Southeastern Cooperative

16 Wildlife Disease Study, University of Georgia, Athens, GA, 30605

\section{Abstract}

18 Farmed American mink (Neovison vison Schreber, 1777) pose a risk to biodiversity owing to escape and release from farms. Feral mink may affect native species in locations where

20 American mink are not endemic, such as Europe. In contrast, escaping domestic mink may

21 hybridize with wild mink in North America, leading to introgression of domestic traits via

22 hybrid-mediated gene flow. We tested this idea in eastern Canada, which has a history of mink 
23 farming. We sampled known domestic and free-ranging mink, and profiled 508 individuals at 15

24 microsatellite loci. We found that $33 \%$ of free-ranging mink were either escaped domestic

25 individuals, domestic-wild hybrids, or were introgressed to domestic or wild parental groups.

26 The greatest prevalence of free-ranging domestic, hybrid, or introgressed mink (59\%) occurred

27 in Nova Scotia, which also had the most mink farms. Historic mink (1980s or earlier) sampled

28 from museums had higher allelic richness and private allelic richness than contemporary wild

29 mink. Domestic mink are artificially selected for traits desired by farmers, and as such,

30 introgression with wild mink may lead to a loss of local adaptation. Our findings demonstrate

31 that continued escape and release of mink could pose risks to the maintenance of genetic

32 integrity in wild mink.

33 Keywords: American mink, domestication, hybridization, insular, introgression, Neovison vison

\section{Introduction}

$$
\text { Domestication is the genetic alteration of a species that leads to an inherited }
$$

predisposition toward humans (Price 1984). Domesticated animals have genomes that differ compared to wild genomes due to artificial selection for certain traits, relaxed selection for other traits, and natural selection to the captive environment. If domesticated animals escape captivity and have an opportunity to interbreed with wild counterparts, a loss of local adaptation can result leading to reduced fitness of hybrid and introgressed offspring (Rhymer and Simberloff 1996). There are many global examples of domestic-wild hybridization and consequent impacts on wild populations. To provide just a few examples, backcrosses between wild and farmed Atlantic salmon (Salmo salar L., 1758) have lower fitness than wild salmon (McGinnity et al. 1993). Cross-breeding between wildcats (Felis sylvestris Schreber, 1777) and 
domestic house cats is a major conservation problem in Europe (Oliviera et al. 2008). Finally, native polecats (Mustela putorius L., 1758) declined to near extinction in Britain after domestic ferrets began to escape and hybridize with the polecats (Davison et al. 1999).

The American mink represents another example of a species where domestic-wild hybridization appears to present a conservation concern. The mink is a North American member of the Mustelidae that has been harvested as a furbearer for centuries. Because of the quality of its fur, and the ease with which it can be raised in captivity, the mink has been domesticated for fur production since the late 1800s. The production of mink on farms expanded greatly beginning in the 1920 s to attain an almost global reach (Joergensen 1985). The global spread of mink farming has brought with it an associated spread of feral American mink, because it is difficult to prevent the escape or intentional release of mink from captivity. Feral mink have colonized many countries around the world including in Europe, Asia, and South America (Medina 1997; Bonesi and Palazon 2007; Zalewski et al. 2011). These escaped domestic mink have led to a variety of biotic impacts on native wildlife, including through predation, competition, and spread of potentially deleterious pathogens (Macdonald and Harrington 2003). Numerous taxa, such as the European mink (Mustela lutreola L., 1761), the water vole (Arvicola amphibious L., 1758), and various ground nesting bird species, appear to have been negatively affected by feral mink (Woodroffe et al. 1990; Maran et al. 1998; Ferreras and Macdonald 1999). Extensive control programs have been deployed in many jurisdictions to limit the impacts and spread of invasive feral mink (Bryce et al. 2011). Nearly all studies about the distribution and biotic effects of feral American mink have taken place in Europe (e.g., Macdonald and Harrington 2003; Bonesi and Palazon 2007; 
67 Zalewski et al. 2011). Presumably, because feral mink are more obvious to detect in locations

where the American mink is exotic, the occurrence of feral American mink in North America, where the mink is native, has not been assessed until recently. Bowman et al. (2007) first showed that the signature of escaping domestic mink could be detected across Canada in wild mink harvest data. Subsequently, studies in Ontario, Canada used genetic methods to document the extent of escaped domestic mink (Kidd et al. 2009; Beauclerc et al. 2013). For example, $78 \%$ of free-ranging mink sampled in the Niagara Falls area were either domestic or domestic-wild hybrids (Kidd et al. 2009).

The biotic effects of feral mink should differ somewhat in North America compared to other locations, owing to the occurrence of native conspecific mink. Sampling in Ontario has shown that escaped domestic mink hybridize with wild mink (Kidd et al. 2009; Beauclerc et al. 2013), which may lead to outbreeding depression due to a loss of local adaptation (Bowman et al. 2007; Beauclerc et al. 2013). An interface for potential pathogen transfer exists when there are lapses in biosecurity on mink farms. Aleutian mink disease virus (AMDV) appears to spread back and forth between mink farms and wild mink (Nituch et al. 2012) and may be spread by escape of infected animals from farms (Nituch et al. 2011). AMDV has also been detected in other free-ranging carnivores, and appears to be transmissible across species, potentially having community-level effects (Farid 2013; Nituch et al. 2015).

Other than the two recent studies in Ontario (Kidd et al. 2009; Beauclerc et al. 2013), there are no studies evaluating the effects of escaping domestic mink on wild mink or other biota in North America. More broadly, there have only been three studies of the population genetic structure of native American mink anywhere in North America (Belliveau et al. 1999; 
Stevens et al. 2005; Beauclerc et al. 2013). Therefore, studies of population genetics of mink in other North American jurisdictions are warranted.

The Canadian Maritimes is a region of eastern Canada that includes Nova Scotia (NS), New Brunswick (NB), and Prince Edward Island (PEI) (Fig. 1). The region has both abundant wild mink populations and prevalent mink farming with an excess of 100 mink producers (Statistics Canada, catalogue 23-013-XIE). Evaluating effects of domestic mink in this area is of particular interest, because NS has the largest mink farming industry in Canada. Bowman et al. (2007) showed that mink harvest by trappers in counties of NS could be predicted by mink farm density, suggesting that mink are escaping from farms and being harvested by trappers. There have been no genetic studies in the Maritimes to evaluate the prevalence of escaped domestic mink, or to investigate the occurrence of domestic-wild hybrids. Furthermore, there have been no studies evaluating the population genetic structure of native mink across the Maritimes.

Therefore, we were interested in assessing both the occurrence and effects of escaped domestic mink and the population structure of native mink. We hypothesized that domestic mink are escaping from farms, becoming feral, and hybridizing with wild mink. We also explored the population genetic structure of wild mink across the Maritimes region.

\section{Materials and methods}

Study area

The Maritimes region of eastern Canada consists of three provinces: PEI (5685 $\left.\mathrm{km}^{2}\right)$, NS $\left(55,284 \mathrm{~km}^{2}\right)$, and NB $\left(72,908 \mathrm{~km}^{2}\right)$. The provinces all have a large amount of coastline with many natural habitats for wild mink. Consequently, wild mink populations have historically been abundant. Both NB and NS are heavily forested, and all three provinces have large 
111 agricultural industries, including mink farms. In 2011, NS had 118 mink farms containing >

112400,000 domestic mink. In the same year, NB had 13 mink farms with an estimated 16,800

113 mink, and PEI had 12 farms with 39,500 domestic mink (Statistic Canada, Table 003-0015). Mink

114 farming has taken place in the region since the early 1900s.

115 Sample collection

Historic mink specimens from NB and NS were sampled in various museums to obtain

117 tissue for DNA analysis (Table 1). Historic samples were from the 1980 s or older. We were able

118 to successfully genotype 9 NS samples and 11 NB samples.

We collected contemporary, free-ranging mink samples from the Maritimes either by

120 collecting frozen, skinned carcasses from commercial, licensed, local fur harvesters during 2008

121 to 2012 , or by sampling pelts prior to auction. We sampled free-ranging mink from NB in 2010

122 by sampling pelts being held for sale at North American Fur Auction (NAFA) in Rexdale, Ontario.

123 We sampled free-ranging mink from PEI at NAFA in 2010 and 2012, and also via a collection of

124 skinned carcasses submitted by licensed trappers to the Canadian Wildlife Health Cooperative

125 in Charlottetown, PEI, opportunistically collected during the 2011-2012 trapping season. We

126 sampled mink in Nova Scotia during 2008 and 2009 through a design where we stratified our

127 sample by mink farm density. We collected free-ranging samples from wild fur harvesters in

128 western NS, which had a high mink farm density, and eastern NS, which had no mink farms (Fig.

129 1; Bowman et al. 2007). We also included 14 free-ranging mink sampled in various NS locations

130 for a previous study by Farid et al. (2010).

We collected known domestic mink from NS via a pelting service, which provided

132 carcasses to us in 2009 following removal of pelts. Pelt colour was difficult to determine 
133 accurately for all of these mink because their pelts had been removed prior to our collection;

134 however, we expected that they were mostly black mink because at the time of sampling, black

135 was the dominant colour line in the Maritime region. We also included in the study for

136 comparative purposes domestic mink genotypes from mink farms in Ontario, for a variety of

137 different colour lines including buff, demi, pastel, iris, and mahogany. These genotypes were

138 the same ones used in an earlier study (see Beauclerc et al. 2013 for Ontario collection details).

139 It is worth noting that domestic mink tend to be line bred within colour types, such that

140 genotyped domestic mink tend to cluster together by colour (Kidd et al. 2009).

141 Laboratory analysis

142 All mink were genotyped using the methods described by Beauclerc et al. (2013). Briefly,

143 approximately $10 \mathrm{mg}$ of muscle was digested and whole genomic DNA extracted using the

144 DNeasy Blood and Tissue Kit (Qiagen Inc., Toronto, ON) according to the manufacturer's

145 directions, or an automated magnetic bead procedure using MagneSil PMPs (Promega,

146 Madison, WI). Purified DNA was quantified with a NanoDrop 8000 or PicoGreen dye (Invitrogen

147 Molecular Probes) with a FLUOStar Galaxy fluorometer (BMG Labtech).

Fifteen microsatellite loci were amplified in eight multiplex polymerase chain reactions

149 (PCR). All reactions were $10 \mathrm{uL}$ and contained $5 \mathrm{ng}$ DNA, $1 \mathrm{X}$ PCR Buffer, $1.5 \mathrm{mM} \mathrm{MgCl} 2,0.2 \mathrm{mM}$

150 each dNTP, $0.2 \mathrm{ug} / \mathrm{mL}$ bovine serum albumin (BSA), and 0.5U Taq polymerase (BioShop Canada

151 Inc., Burlington, ON, Canada). Thermal cycling used an Eppendorf Mastercycler Pro thermal

152 cycler with an initial denaturation of $94^{\circ} \mathrm{C}$ for $5 \mathrm{~min}$, followed by 30 cycles of denaturation at

$15394^{\circ} \mathrm{C}$ for $30 \mathrm{sec}$, annealing for $1 \mathrm{~min}$, and extension at $72^{\circ} \mathrm{C}$ for $1 \mathrm{~min}$, and a final extension of

$15460^{\circ} \mathrm{C}$ for $45 \mathrm{~min}$. Reactions were pooled into 3 groups (Table 2) with GeneScan 500 ROX for 
155

156

157

158

159

160

161

162

163

164

165

166

167

168

169

170

171

172

173

174

175

176

genotyping on an $\mathrm{ABI} 3730$ automated sequencer. Fragment sizes were scored using

GeneMapper 4.0 (Applied Biosystems). See Beauclerc et al. (2013) for additional details

including primer concentrations and sources, annealing temperatures, and multiplex conditions.

DNA from historic samples was extracted in a laboratory dedicated to processing ancient DNA at Trent University in Peterborough, Ontario. Fresh reagents and supplies were used at all stages. Samples were extracted with the Qiagen DNeasy Tissue kit according to the manufacturer's instructions, with blanks incorporated for every 10 samples. PCR was carried out with the same conditions as the contemporary samples, except 50 cycles were used for the denaturing, annealing, and extension steps.

Statistical analysis

Individuals with 10 or more scored loci were used for analyses. The program MicroChecker v. 2.2.3 (van Oosterhout et al. 2004) was used to check for genotyping errors, large allele dropout, and the presence of null alleles. Significant deviations from Hardy-Weinberg equilibrium (HWE) and linkage disequilibrium (LD) were calculated in GenePop on the Web v.4.2 (Rousset 2008). For HWE, the estimation of exact $p$-values for deficiency of heterozygotes and $\mathrm{F}_{\mathrm{IS}}$ (Weir and Cockerham 1984) was conducted using a Markov chain method with 5000 dememorization steps, 1000 batches, and 5000 iterations each. For linkage disequilibrium, a log likelihood ratio statistic was used. Summary statistics (number of alleles, allele frequencies, observed and expected heterozygosities, and pairwise population differentiation $\left(F_{S T}\right)$ ) were estimated using GenAIEx v. 6.5 (Peakall and Smouse 2012). Mean allelic richness per locus as well as private allelic richness were calculated by applying the rarefaction method in HP-Rare 
177 v.1.1 (Kalinowski 2005) to account for uneven sample sizes (Kalinowski 2004). Allelic richness

178 was calculated for sampling locations (i.e., pre-defined groups), historic samples from Nova

179 Scotia and New Brunswick as well as for clusters identified using $K=5$ during the Bayesian 180 assignment analysis described below.

To assess the most probable number of inferred genetic population clusters $(\mathrm{K})$ and assign individuals to their likely population of origin, the program Structure v. 2.3.3 (Pritchard et al. 2000) was run on a high-performance computing cluster using the admixture model with correlated allele frequencies (Falush et al. 2003). Five simulations for each value of $K$ from 1 to 10 were run using a burn-in of $10^{6}$ and $\mathrm{MCMC}$ chain of $10 \times 10^{6}$ iterations without prior geographical information. The program Structure Harvester v. 0.6.93 (Earl and vonHoldt 2012) was used to summarize Structure runs and determine the most likely number of clusters based on $\Delta K$ (Evanno et al. 2005). Bar plots of individual membership coefficients $(q)$ for $K=2$ and $K=$ 5 were generated with Distruct v.1.1 (Rosenberg 2004). An individual was assigned to a single cluster if it had a $q>0.8$, or jointly to two or more clusters such that the minimum sum of $q_{i}+q_{j}$

$+\ldots q_{\mathrm{n}} \geq 0.80$ for admixed individuals, thereby ensuring that at least $80 \%$ of an individual's genome was assigned to the inferred cluster(s) (Verardi et al. 2006).. This threshold was based on a simulation analysis undertaken by Kidd et al. (2009) in which $q>0.8$ was shown to be of sufficient power to correctly assign $96 \%$ of mink to either domestic, wild, or hybrid groups. We used a second clustering method to confirm patterns observed in the Structure 196 analysis. We used adegenet v2.0.1 (Jombart 2008) to perform a Principal Component Analysis 197 (PCA), and we then inspected the distribution of domestic, wild, and hybrid individuals (as identified using Structure) on the PCA biplot. 
We also conducted an assignment test with Structure using only the individuals assigned

200

201

202

203

204

205

206

207

208

209

210

211

212

213

214

215

216

217

218

219

220

as wild $\left(q_{\text {wild }} \geq 0.80\right)$ in the initial Structure analysis. We did this to observe whether there was any substructure across the study area among wild mink. As in the first assignment test with the full dataset, we used Structure v. 2.3.3 (Pritchard et al. 2000) for the analysis of wild mink.

Finally, using the Structure results from the full dataset for $K=5$, we grouped individuals for which $q \geq 0.8$, and removed all hybrid individuals. We treated the historic specimens as a separate group, regardless of their assignment in Structure. We then constructed a neighbourjoining tree for these 6 groups using genetic distance $\left(D_{A}\right)$ in Poptree 2 (Takezaki et al. 2010). Significance was assessed using 1000 bootstrap replications.

\section{Results}

We sampled and successfully genotyped 173 mink from farms in NS, and 133 mink of various colour lines from Ontario, including mahogany, pastel, demi, buff, and iris. We genotyped 13 free-ranging mink from NB, 84 from NS, and 85 from PEI. In addition, we genotyped 20 historic Maritime mink samples, varying in year of origin from 1894 to 1983 (Table 1). Overall, we evaluated 508 genotyped mink samples in the study.

All 15 loci were polymorphic with 4-17 alleles each (Table 2). In total, 150 tests for HWE were performed and of these, 50 showed a significant deviation from expected proportions. After Bonferroni correction ( $\alpha=0.05 / 150 \leq 0.0003), 12$ tests remained significant, which were due to heterozygote deficiencies. Accordingly, $F_{I S}$ values per population revealed a similar pattern with values ranging from $0.018-0.17$ (Table 3). Linkage disequilibrium (LD) was found in 130 comparisons at the population level. However, only 23 loci pairs deviated significantly from LD after Bonferroni correction. Notably, 16 of these linkage pairs were found in free- 
221 ranging mink from Nova Scotia. There were signs of null alleles in 26 of 150 comparisons, but

222 none of the loci showed null alleles in a majority of sampled populations. Null allele proportions

223 ranged from $4-28 \%$.

224 Structure analysis

225

Following the Evanno method (Evanno et al. 2005), maximal $\Delta K$ was found at $K=2$,

where domestic mink were differentiated from wild mink. Additional differentiation may be

227 present at higher $K$ values however, so we also studied the assignment results for $K=5$, which was found in a previous study (Beauclerc et al. 2013). We found four domestic groups (black, brown, iris, and mahogany), along with a fifth group that we perceived to be a wild genotype, consisting of free-ranging mink from all three Maritime provinces (Fig. 2). Of all free-ranging Maritime mink $(n=182), 26.9 \%$ were identified as farm escapees $(n=$

$\left.23249, q_{\text {wild }}<0.20\right)$ while $6.0 \%(n=11)$ were either domestic-wild hybrids or backcrosses $\left(q_{\text {wild }} 0.20\right.$ 0.80). Importantly, of all farm escapees and hybrids, 41 (83.7\%) and 6 (54.5\%) were found in NS (PEI 16.3\% and 36\%; NB 0\% and 9.1\%, respectively). Overall, 59.5\% of free-ranging mink sampled in NS were either domestic escapees, domestic-wild hybrids, or introgressed animals. The mean membership coefficient $(q)$ of free-ranging Maritime mink in the wild group was 0.66 ,

237 and in the black group was 0.21. Free-ranging mink from NS had a low membership coefficient 238 to the wild group of 0.44 . Notably, free-ranging mink from NS had only a slightly lower 239 membership coefficient in the black group (0.37). Thus, free-ranging mink from NS consisted of 240 a mixture of black farm escapees, introgressed and hybrid mink, and wild mink. Introgression in 241 the NS samples appeared to be greater in the direction of domestic parentals, as indicated by 242 the transition in wild assignment probabilities between low (0.2) and moderate (0.5) values 
243 (Fig. 2). There were more individuals with probabilities between 0.2 and $0.5(n=5)$ then there 244 were between 0.5 and $0.8(n=1)$. At $K=5$, brown, black, iris, and mahogany individuals had 245 high mean membership coefficients to their respective groups (Table 4). In contrast, individuals

246 from NS mink farms had relatively lower mean membership to the black (0.58) and mahogany 247 (0.34) groups, indicating that our domestic mink samples from NS consisted of both black and 248 mahogany individuals. As with the $K=2$ analysis, free-ranging Maritime mink did not show any 249 significant substructure among sampling locations (NS, NB, PEI) at $K=5$. Historic mink had a 250 higher membership to the wild group than did contemporary free-ranging mink samples (Table $2514)$. Principal components analysis supported our Structure assignments for $K=2$, as there 253 was generally a good separation between domestic and wild mink (as identified using Structure) 254 in the PCA biplot, and hybrid mink tended to be intermediate (Fig. 3). The separation between 255 wild and domestic mink occurred mostly along the first principal component (PC1). When we considered only mink identified as wild in our analysis of the full dataset, we 257 found evidence for some substructure of wild mink. An assignment test of these wild mink 258 demonstrated that $K=2$, where PEI mink were assigned as a distinct group separated from 259 mainland NS and NB mink (Structure plot not shown). The mean (SE) assignment probabilities 260 to their respective groups were $0.977(0.006)$ for PEI and $0.954(0.009)$ for the mainland (NS 261 and NB). Some separation between PEI and mainland mink was also apparent from the PCA 262 biplot, appearing as a split between wild mink along PC2 (Fig. 3).

\section{Genetic diversity}


The historic samples mostly assigned with the wild group, although three individuals

265

266

267

268

269

270

271

272

273

274

275

276

277

278

279

280 281 (Fig. 4).

282

284

285

from NB were assigned as domestic-wild hybrids. Historic samples did however, have both the highest allelic richness, and the highest private allelic richness. Likewise, wild mink showed higher (private) allelic richness when compared to different farm mink varieties (Table 5).

Further, wild mink showed highest observed and expected heterozygosity values and therefore, the highest genetic diversity of all groups compared. Within domestic mink, mahogany animals had the highest genetic diversity (Table 5), indicated by the greatest average number of alleles, $A_{R}, A_{p r}$ and $H_{E}$, which is probably consistent with their hybrid origin. Mahogany mink are a result of mixing brown and black lines (Kidd et al. 2009). This may also explain the fairly high $F_{I S}$ value for the mahogany group as a Wahlund effect was likely present due to the admixture of two different populations.

Black and iris were the least differentiated of the $K=5$ groups according to pairwise estimates of $F_{S T}$ (Table 6). Conversely, wild mink were the most differentiated of all the groups, with a mean $F_{S T}$ compared to the 4 other groups of 0.081 (Table 6). A neighbour joining network based on allele frequencies for the $K=5$ groups, with the historic mink included as a separate, sixth group, demonstrated that historic mink were the most distant from the domestic mink, whereas wild mink were intermediate between the 4 domestic groups and the historic group

\section{Discussion}

Based on genetic data, our study confirmed that domestic mink have either been escaping, or have been released, from mink farms in the Canadian Maritimes and are hybridizing with wild American mink. We found that $26.9 \%$ of 182 free-ranging mink sampled 
286

287

288

289

290

291

292

293

294

295

296

297

298

299

300

301

302

303

304

305

were domestic escapees, and a further $6.0 \%$ were domestic-wild hybrids. The impact of mink farming appeared greatest in NS, which contained $>80 \%$ of the escaped domestic mink that we sampled. This is not surprising, insofar as NS had the largest mink farming industry in Canada at the time of our study. Nevertheless, escaped domestic or hybrid mink were detected in all three Maritime provinces. The prevalence of escaped domestic and hybrid mink in the Maritimes is similar to the escapee and hybrid prevalence ( $13 \%$ and $5 \%$, respectively) measured across an Ontario study area (Beauclerc et al. 2013). It is notable however, that in both situations, impacts of domestic mink, such as hybridization, appear to be greater at sites in close proximity to mink farms. In Beauclerc et al.'s (2013) study, southwestern Ontario had the highest mink farm density, and $28 \%$ of free-ranging mink sampled in that area were domesticwild hybrids, while $36 \%$ were domestic escapees. NS had the highest prevalence in the Maritimes of domestic escapees (51\% of free-ranging mink sampled) and domestic-wild hybrids (8\%). The proximate relationship between mink farms and the impacts of domestic mink is consistent with the idea that feral domestic mink have low fitness in a North American context, which may help to limit the spatial extent of their impacts on native biodiversity (Nituch et al. 2011; Beauclerc et al. 2013). The idea that escaped domestic mink in North America have low fitness has not been directly tested however. It is clear that feral mink are capable of exhibiting high fitness in non-native contexts (Macdonald and Harrington 2003). It is also important to note that various effects of domestic mink such as the spread of pathogens and the diffusion of genes can have widespread impacts (e.g., Nituch et al. 2012, 2015).

Escaped domestic mink can have deleterious effects on native biota through a number of mechanisms. Genetic diversity in wild mink may be reduced by escaped domestic mink 
308 because domestic mink show lower genetic diversity than wild mink as the comparison to 309 historic samples indicated. Even if hybrid mink have low fitness, hybridization may have ongoing consequences on wild mink due to the continuous presence of free-ranging domestic

311 mink on the landscape over many generations. Hybridization and introgression of domestic

312 traits into the wild mink population could lead to outbreeding depression and reduced fitness

313 through loss of local adaptation and co-adapted gene complexes (McGinnity et al. 2003). In

314 addition, mink farms may serve as point sources of pathogens such as AMDV and escaping

315 domestic mink may spread AMDV to wild mink and other species such as striped skunks

316 (Mephitis mephitis Schreber, 1776) and short-tailed weasel (Mustela erminea L., 1758) (Nituch

317 et al. 2011, 2012, 2015; Farid 2013). Domestic mink may also have impacts on native biota

318 through predation and competition (Macdonald and Harrington 2003; Bonesi and Palazon

319 2007). For example, predation by feral American mink appears to have reduced abundance of

320 the water vole (Jefferies et al. 1989), a species which is now of conservation concern in some

321 European jurisdictions. Competition between feral mink and European mink is one hypothesis

322 for the cause of endangerment of the European species (Maran and Henttonen 1995).

Wild mink had the highest private allelic richness, and some of this appeared

324 attributable to historic mink, which had the highest private allelic richness of any sampled

325 group. We observed the presence of two private alleles in our historic samples (Table 1) that

326 did not appear in any other samples. Between our study and the earlier work of Beauclerc et al.

327 (2013), we have now identified five private alleles from 41 individual mink samples obtained

328 from historic collections in museums. While it is possible that these missing alleles are due to

329 sampling or genetic drift, we must also consider that they represent a loss of genetic diversity 
330 due to reduced population size or even due to the effects of introgression with domestic mink.

331 The historic samples had the highest membership coefficient to the wild group of all our

332 samples, including the contemporary free-ranging animals (0.85 vs 0.66$)$. Overall, the wild mink

333 group had high allelic richness and high expected heterozygosity compared to various domestic

334 colour lines, (although mahogany mink had the highest allelic richness). Domestic mink

335 generally had lower values for measures of genetic diversity than did wild mink in our study,

336 which was not surprising given that domestic mink are line bred. This contrasts with work of

337 Belliveau et al. (1999) who found that wild mink trapped from one area in NB had a lower

338 expected heterozygosity than various lines of domestic mink, although this may have been

339 attributable to the small geographic area sampled for wild mink (Belliveau et al. 1999).

We observed weak evidence of spatial genetic structure among contemporary wild mink

341 in the Maritimes. All Structure analyses of the full dataset suggested a single group containing

342 all of the presumed wild mink. There was some substructure that emerged when we assessed

343 only mink with a wild assignment probability, suggesting that mink in PEI are somewhat distinct

344 from mink on the mainland. The extensive network of waterways available to mink in the

345 Maritimes suggests that populations of this semi-aquatic animal should be well connected

346 throughout the region (e.g., Stephens et al. 2005; Laurence at el. 2013), and that is generally

347 what we observed; however, it seems that the Northumberland Strait separating PEI from the

348 mainland may be somewhat of a barrier to mink. We were not able to test for isolation-by-

349 distance (IBD) due to the low accuracy of spatial locations associated with some of the samples

350 we obtained from trappers. IBD was not observed in wild mink over a relatively similar spatial

351 extent in Ontario (Beauclerc et al. 2013). It may be worthwhile comparing the genotypes of 
352 Maritime mink to those from other regions of North America to evaluate whether there is 353 extensive panmixia, as has been observed in some similar species such as the marten (Kyle and 354 Strobeck 2002).

The prevalence of mink escaping or being released from farms suggests that more attention should be paid to biosecurity, both to prevent mink from escaping and people from

357 accessing mink farms. In all Canadian jurisdictions where this topic has been studied, there is 358 evidence of free-ranging domestic, hybrid, or introgressed mink. It is notable that European studies have also shown domestic mink to escape and hybridize with long-established feral mink (e.g., Zalewski et al. 2011), suggesting that the escape of farmed mink is a widespread 361 process with an extensive footprint. In Canada, biosecurity on farms tends to be a provincial 362 responsibility, and measures vary by province. Available measures to reduce risk of escape or 363 release include establishing minimum fencing standards, and licensing of mink farmers.

\section{Conservation implications}

Our analysis demonstrated that free-ranging domestic mink are present in the

Maritimes region of Canada. This is consistent with previous indications that feral domestic

367 mink in NS supplement the wild mink harvest (see Fig. 3 in Bowman et al. 2007). Domestic

368 mink occurred throughout the region, but were most prevalent in close proximity to mink

369 farms. The findings of our study are also consistent with earlier work from Ontario that found

370 prevalent domestic-wild hybridization in close proximity to mink farms. The long term

371 implications of escaped domestic mink are unknown, although an analysis of harvest data

372 across the country suggested that mink farms were associated with population declines in wild

373 mink (Bowman et al. 2007). A variety of mechanisms are possible that could lead to declines in 
374 wild mink, including outbreeding depression due to hybridization and introgression with

375 domestic mink, and increased spread of pathogens such as AMDV (Bowman et al. 2007, Kidd et

376 al. 2009). The reduced number of alleles in contemporary vs historic mink is also consistent

377 with the idea that there has been a loss of genetic diversity in wild mink, possibly due to

378 introgression with domestic animals. We suggest that mink farm biosecurity should be an

379 important priority for jurisdictions with mink farms, to mitigate potential effects of escaping

380 domestic mink on wild mink.

381 Acknowledgments

382 Funding was provided by: NSERC Strategic Projects grant to AISH and JB, NSERC Discovery

383 Grants to AISH and JB, Prince Edward Island Wildlife Conservation Fund, and the Ontario

384 Ministry of Natural Resources and Forestry. We thank the New Brunswick Museum, the Acadia

385 University Museum, the Nova Scotia Museum, and the Canadian Museum of Nature for

386 providing samples, and we thank the Canadian Wildlife Health Cooperative and the many

387 trappers and mink farmers who assisted us in the study.

\section{References}

Beauclerc, K., Bowman, J., and Schulte-Hostedde, A.I. 2013. Assessing the cryptic invasion of a domestic conspecific: American mink in their native range. Ecol. Evol. 3: 2296-2309. doi: 10.1002/ece3.630.

Belliveau, A.M., Farid, A., O’Connell, M., and Wright, J.M. 1999. Assessment of genetic variability in captive and wild American mink (Mustela vison) using microsatellite markers. Can. J. Anim. Sci. 79: 7-16. doi: 10.4141/A97-115.

Bonesi, L., and Palazon, S. 2007. The American mink in Europe: status, impacts, and control. Biol. Conserv. 134: 470-483. doi: 10.1016/j.biocon.2006.09.006.

Bowman, J., Kidd, A.G., Gorman, R.M., and Schulte-Hostedde, A.I. 2007. Assessing the potential for impacts by feral mink on wild mink in Canada. Biol. Conserv. 139: 12-18. doi: 10.1016/j.biocon.2007.05.020. 
403

404

405

406

407

408

409

410

411

412

413

414

415

416

417

418

419

420

421

422

423

424

425

426

427

428

429

430

431

432

433

434

435

436

437

438

439

440

441

442

443

444

Bryce, R., Oliver, M.K., Davies, L., Gray, H., Urquhart, J. et al. 2011. Turning back the tide of American mink invasion at an unprecedented scale through community participation and adaptive management. Biol. Conserv. 144: 575-583. doi: 10.1016/j.biocon.2010.10.013.

Davison, A., Birks, J.D.S., Griffiths, H.I., Kitchener, A.C., Biggins, D. et al. 1999. Hybridization and the phylogenetic relationship between polecats and domestic ferrets in Britain. Biol. Conserv. 87: 155-161. doi: 10.1016/S0006-3207(98)00067-6.

Earl, D.A., and vonHoldt, B.M. 2012. STRUCTURE HARVESTER: a website and program for visualizing STRUCTURE output and implementing the Evanno method. Conserv. Genet. Resour. 4: 359-361. doi: 10.1007/s12686-011-9548-7.

Evanno, G., Regnaut, S., and Goudet, J. 2005. Detecting the number of clusters of individuals using the software STRUCTURE: a simulation study. Mol. Ecol. 14: 2611-2620. doi: 10.1111/j.1365-294X.2005.02553.x.

Falush, D., Stephens, M., and Pritchard, J.K. 2003. Inference of population structure: extensions to linked loci and correlated allele frequencies. Genetics, 164: 1567-1587.

Farid, A.H., Rupasinghe, P., Mitchell, J.L., and Rouvinen-Watt, K. 2010. A survey of Aleutian mink disease virus infection of feral American mink in Nova Scotia. Can. Vet. J. 51: 75-77.

Farid, A.H. 2013. Aleutian mink disease virus in furbearing mammals in Nova Scotia, Canada. Acta Veterinaria Scandinavica 55:10. doi: 10.1186/1751-0147-55-10.

Ferreras, P., and MacDonald, D.W. 1999. The impact of American mink Mustela vison on water birds in the upper Thames. J. Appl. Ecol. 36:701-708. doi: 10.1046/j.13652664.1999.00431.x.

Jefferies, D. J., Morris, P. A., and Mulleneux, J. E. 1989. An enquiry into the changing status of the water vole Arvicola terrestris in Britain. Mammal Rev. 19: 111-131.

Joergensen, G. 1985. Mink production. Scientifur, Denmark.

Jombart, T. 2008. adegenet: a R package for the multivariate analysis of genetic markers. Bioinformatics, 24: 1403-1405. doi: 10.1093/bioinformatics/btn129.

Kalinowski, S.T. 2004. Counting alleles with rarefaction: private alleles and hierarchical sampling designs. Conserv. Genet. 5: 539-54.doi: 10.1023/B:COGE.0000041021.91777.1a.

Kalinowski, S.T. 2005. HP-Rare: a computer program for performing rarefaction on measures of allelic diversity. Mol. Ecol. Notes 5: 187-189. doi: 10.1111/j.1471-8286.2004.00845.x. 
Kidd, A.G., Bowman, J., Lesbarrères, D., and Schulte-Hostedde, A.I. 2009. Hybridization between escaped domestic and wild American mink (Neovison vison). Mol. Ecol. 18: 1175 - 1186. doi: 10.1111/j.1365-294X.2009.04100.x.

Kyle, C.J., and Strobeck, C. 2002. Genetic homogeneity of Canadian mainland marten populations underscores the distinctiveness of Newfoundland pine martens (Martes americana atrata). Can. J. Zool. 81: 57-66. doi: 10.1139/z02-223.

Laurence, S., Smith, M.J., and Schulte-Hostedde, A.I. 2013. Effects of structural connectivity on fine scale population genetic structure of muskrat, Ondatra zibethicus. Ecology and Evolution. 3: 3524-3535. doi: 10.1002/ece3.741.

Macdonald, D.W., and Harrington, L.A. 2003. The American mink: the triumph and tragedy of adaptation out of context. N.Z. J. Zool. 30: 421-441. doi: 10.1080/03014223.2003.9518350.

Maran, T. and Henttonen, H. 1995. Why is the European mink, Mustela lutreola disappearing? A review of the process and hypotheses. Ann. Fenn. Zool. 32: 47-54.

Maran, T., MacDonald, D.W., Kruuk, H., Sidorovich, V., and Rozhnov, V.V. 1998. The continuing decline of the European mink Mustela lutreola: evidence for the intraguild aggression hypothesis. In Behaviour and ecology of riparian mammals. Edited by N. Dunstone, and M. L. Gorman. Cambridge University Press, Cambridge, United Kingdom. pp. 297-232.

McGinnity, P., Prodöhl, P., Ferguson, A., Hynes, R., Ó Maoiléidigh, N., et al. 2003. Fitness reduction and potential extinction of wild populations of Atlantic salmon, Salmo salar, as a result of interactions with escaped farm salmon. Proc. R. Soc. Lond. B Biol. Sci. 270: 2443-2450. doi: 10.1098/rspb.2003.2520.

Medina, G. 1997. A comparison of the diet and distribution of southern river otter (Lontra provocax) and mink (Mustela vison) in southern Chile. J. Zool. (Lond.) 242: 291-297. doi: 10.1111/j.1469-7998.1997.tb05802.x.

Nituch, L.A., Bowman, J., Beauclerc, K.B., and Schulte-Hostedde, A.I. 2011. Mink farms predict Aleutian disease exposure in wild American mink. PLoS ONE, 6:e21693. doi: 10.1371/journal.pone.0021693.

Nituch, L.A., Bowman, J., Wilson, P., and Schulte-Hostedde, A.I. 2012. Molecular epidemiology of Aleutian disease virus in free-ranging domestic, hybrid, and wild mink. Evol. Appl. 5: 330-340. doi: 10.1111/j.1752-4571.2011.00224.x.

Nituch, L. A., Bowman, J., Wilson, P.J., and Schulte-Hostedde, A.I. 2015. Aleutian mink disease virus in striped skunks: evidence for cross-species spillover. J. Wildl. Dis. 51: 389-400. doi: 10.7589/2014-05-141. 
489

490

491

492

493

494

495

496

497

498

499

500

501

502

503

504

505

506

507

508

509

510

511

512

513

514

515

516

517

518

519

520

521

522

523

524

525

526

527

528

529

530
Oliviera, R., Godinho, R., Randi, E., and Alves, P.C. 2008. Hybridization versus conservation: are domestic cats threatening the genetic integrity of wildcats (Felis silvestris silvestris) in Iberian Peninsula? Philos. Trans. R. Soc. Lond. B Biol. Sci. No. 363: 2953-2961. doi: 10.1098/rstb.2008.0052.

Peakall, R., and Smouse, P.E. 2012. GenAIEx 6.5: genetic analysis in Excel. Population genetic software for teaching and research - an update. Bioinformatics, 28: 2537-2539. doi: 10.1093/bioinformatics/bts460.

Price, E.O. 1984. Behavioral aspects of animal domestication. Q. Rev. Biol. 59: 1-31.

Pritchard, J.K., Stephens, M., and Donnelly, P. 2000. Inference of population structure using multilocus genotype data. Genetics, 155:945-959.

Rhymer, J.M., and Simberloff, D. 1996. Extinction by hybridization and introgression. Annu. Rev. Ecol. Syst. 27: 83-109.

Rosenberg, N.A. 2004. Distruct: a program for the graphical display of population structure. Mol. Ecol. Notes 4:137-138. doi: 10.1046/j.1471-8286.2003.00566.x.

Rousset, F. 2008. GenePop'007: a complete re-implementation of the GenePop software for Windows and Linux. Mol. Ecol. Resour. 8:103-106. doi: 10.1111/j.1471-8286.2007.01931.x.

Stevens, R.T., Kennedy, M.L., and Kelley, V.R. 2005. The genetic structure of American mink (Mustela vison) populations. Southwestern Nat. 50: 350-355.

Takezaki, N., Nei, M., and Tamura, K. 2010. POPTREE2: software for constructing population trees from allele frequency data and computing other population statistics with Windowsinterface. Mol. Biol. Evol. 27: 747-752.

van Oosterhout, C., Hutchinson, W.F., Wills D.P., and Shipley, P. 2004. Micro-Checker: software for identifying and correcting genotyping errors in microsatellite data. Mol. Ecol. Resour. 4: 535-538. doi: 10.1111/j.1471-8286.2004.00684.x.

Verardi A., Lucchini V., and Randi, E. 2006. Detecting introgressive hybridization between freeranging domestic dogs and wild wolves (Canis lupus) by admixture linkage disequilibrium analysis. Mol. Ecol. 15: 2845-2855. doi:10.1111/j.1365-294X.2006.02995.x.

Weir, B. S., and Cockerham, C. C. 1984. Estimating F-statistics for the analysis of population structure. Evolution, 38: 1358-1370. 
531 Woodroffe, G., Lawton, J., and Davidson, W. 1990. The impact of feral mink Mustela vison on 532 water voles Arvicola terrestris in the North Yorkshire Moors National Park. Biol. Conserv. 51:4953362.

534 Zalewski, A., Michalska-Parda, A., Ratkiewicz, M., Kozakiewicz, M., Bartoszewicz, M., et al. 535 2011. High mitochondrial DNA diversity of an introduced alien carnivore: comparison of feral 536 and ranch American mink Neovison vison in Poland. Divers. Distrib. 17: 757-768. doi:

537 10.1111/j.1472-4642.2011.00767.x. 
539 Figure 1. Study area in eastern Canada where both domestic and free-ranging American mink 540 (Neovison vison Schreber, 1777) were sampled for a study of population genetics. Free-ranging 541 mink were sampled from New Brunswick $(n=13)$, Nova Scotia $(n=84)$ and Prince Edward Island 542 ( $n=85)$. In addition, historic mink samples from the region were sampled in museums $(n=20)$, 543 and mink were genotyped from farms in Nova Scotia $(n=173)$. Most of the free-ranging mink in 544 Nova Scotia were sampled in strata that were either close (open symbol) or far (closed symbol) 545 from the region with extensive mink farming.

546 Figure 2. Bar plots for population structure analysis and identification of admixed American 547 mink (Neovison vison Schreber, 1777) individuals using Structure v.2.3.3 (Pritchard et al. 2000) 548 at $K=2(\mathrm{a})$ and $K=5$ (b). Individuals are grouped by known sampling locations and/or colour 549 phase ( $X$ axis) separated by black vertical lines. Each coloured vertical bar represents one 550 individual and different colours show group membership coefficients $(q) . K=2$ discriminated 551 domestic mink (red) from wild minks (green) whereas $K=5$ identified wild mink (green) as a 552 separate group and additionally differentiated according to fur colour within domestic farm 553 mink (black mink = red; brown mink = yellow; iris mink = orange; mahogany mink = blue). NBW $554=$ New Brunswick free-ranging mink, NSW = Nova Scotia free-ranging mink, PEI = Prince Edward 555 Island free-ranging mink, and NSF = Nova Scotia domestic mink.

556 Figure 3. Biplot from a Principal Components Analysis (PCA) comparing microsatellites from 557 American mink (Neovison vison Schreber, 1777) with different assignments (domestic, wild, or 558 hybrid) based on a Structure analysis.

559 Figure 4. A neighbour joining network based on allele frequencies for the five genetic groups of 560 American mink (Neovison vison Schreber, 1777) identified in a study of mink population 561 genetics in the Canadian Maritimes. 
Table 1. Historic American mink (Neovison vison Schreber, 1777) sampled in a study of population genetics of mink in the Canadian Maritimes ${ }^{1}$.

\begin{tabular}{|c|c|c|c|c|c|}
\hline ID & Sex & Prov & Site description & Year & Museum \\
\hline AM ND 2 & & & & & Acadia University Museum \\
\hline CMNA 4257 & $\mathrm{~F}$ & NB & Miramichi Rd, 15 mi. from Bathurst & 1921 & Canadian Museum of Nature \\
\hline CMNA 4197 & M & NB & Miramichi Rd, 15 mi. from Bathurst & 1921 & Canadian Museum of Nature \\
\hline FMNH 5454 & M & NB & Trousers Lake & 1894 & Field Museum of Natural History \\
\hline NBM 1340 & M & NB & Sunbury Co.; Maugerville & 1975 & New Brunswick Museum \\
\hline NBM 2172 & $M$ & NB & York Co; Fredricton & 1958 & New Brunswick Museum \\
\hline NBM 2776 & $\mathrm{~F}$ & NB & Saint John Co.; Foster Thurston Drive & 1983 & New Brunswick Museum \\
\hline NBM 5456 & $M$ & NB & York Co; Mactaquac & 1975 & New Brunswick Museum \\
\hline NBM $5941^{2}$ & $M$ & NB & Charlotte Co.; Campobello Island & 1894 & New Brunswick Museum \\
\hline NBM 890 & M & NB & Charlotte Co.; Pocologan & 1971 & New Brunswick Museum \\
\hline NBM 926 & $\mathrm{~F}$ & NB & York Co.; Ayers Lake & 1970 & New Brunswick Museum \\
\hline NBM 4550 & M & NB & Newcastle & 1965 & New Brunswick Museum \\
\hline CMNA 12754 & & NS & Nipisiquit River near Bathurst & 1935 & Canadian Museum of Nature \\
\hline CMNA 32731 & M & NS & Cape Breton Co. (NS); Louisburg & 1962 & Canadian Museum of Nature \\
\hline NSM 10706 & $\mathrm{~F}$ & NS & & & Nova Scotia Museum \\
\hline NSM 10707 & & NS & Halifax Co.; Spryfield & 1963 & Nova Scotia Museum \\
\hline NSM 10708 & & NS & Cumberland Co.; Halfway River & 1977 & Nova Scotia Museum \\
\hline NSM 972.312.001 & $\mathrm{F}$ & NS & Halifax Co.; Oathill Lake, Dartmouth & 1919 & Nova Scotia Museum \\
\hline NSM 972.312.014 & & NS & Lunenburg Co.; LaHave River & 1921 & Nova Scotia Museum \\
\hline NSM 976.037.000 & & NS & Inverness Co.; Whycocomagh & 1976 & Nova Scotia Museum \\
\hline
\end{tabular}

${ }^{1} \mathrm{NB}=$ New Brunswick; NS = Nova Scotia; ${ }^{2}$ Collection date and sex for this sample are not firm. 
Table 2. Summary statistics for microsatellite loci used in a study of American mink (Neovison vison Schreber, 1777) in the Canadian Maritimes. Allele size ranges are shown, along with number of alleles $\left(\mathrm{N}_{\mathrm{A}}\right)$, observed heterozygosity $\left(\mathrm{H}_{\mathrm{O}}\right)$, expected heterozygosity $\left(\mathrm{H}_{\mathrm{E}}\right)$, and probability of deviations from Hardy-Weinberg Equilibrium (HWE $p$ ).

\begin{tabular}{|c|c|c|c|c|c|}
\hline Locus & Range $^{1}$ & $\mathrm{~N}_{\mathrm{A}}$ & $\mathrm{H}_{\mathrm{O}}$ & $\mathrm{H}_{\mathrm{E}}$ & HWE $p$ \\
\hline \multicolumn{6}{|c|}{ Multiplex 1} \\
\hline Mvi111 & $84-110$ & 9 & 0.663 & 0.762 & $>0.0001$ \\
\hline Mvi1006 & $136-168$ & 14 & 0.652 & 0.806 & $>0.0001$ \\
\hline Mvi1272 & $163-181$ & 10 & 0.707 & 0.819 & $>0.0001$ \\
\hline Mvi99 & $316-356$ & 17 & 0.730 & 0.830 & $>0.0001$ \\
\hline Mvi4001 & $223-233(221)$ & 7 & 0.580 & 0.615 & 0.0267 \\
\hline Mvi114 & $62-84(86)$ & 12 & 0.670 & 0.768 & $>0.0001$ \\
\hline Mvi1302 & $207-221(201)$ & 9 & 0.475 & 0.785 & $>0.0001$ \\
\hline \multicolumn{6}{|c|}{ Multiplex 2} \\
\hline Mvi1016 & $216-236$ & 11 & 0.756 & 0.815 & $>0.0001$ \\
\hline Mvi1321 & $88-108$ & 11 & 0.657 & 0.807 & $>0.0001$ \\
\hline Mvi2243 & $123-155$ & 10 & 0.608 & 0.704 & $>0.0001$ \\
\hline \multicolumn{6}{|c|}{ Multiplex 3} \\
\hline Mvi1354 & $176-198$ & 12 & 0.590 & 0.764 & $>0.0001$ \\
\hline Mvi002 & $176-188$ & 4 & 0.126 & 0.131 & 0.0023 \\
\hline Mvi072 & $255-269$ & 7 & 0.517 & 0.621 & $>0.0001$ \\
\hline Mvi1342 & $136-174$ & 16 & 0.684 & 0.788 & $>0.0001$ \\
\hline Mvi075 & $111-131$ & 10 & 0.759 & 0.831 & $>0.0001$ \\
\hline
\end{tabular}

${ }^{1}$ Alleles in brackets were found only in historic samples originating from museums. 
Table 3. Summary statistics for microsatellite loci at the level of sampled populations, used in a study of American mink (Neovison vison Schreber, 1777) in the Canadian Maritimes. Number of individuals in each group $(N)$ are shown, along with number of alleles $\left(N_{A}\right)$, allelic richness $\left(A_{R}\right)$, number of private alleles $\left(N_{A P}\right)$, private allelic richness $\left(A_{R P}\right)$, observed heterozygosity $\left(H_{O}\right)$, expected heterozygosity $\left(H_{E}\right)$, standard error $(S E)$ of $H_{E}$, and probability of deviations from Hardy-Weinberg Equilibrium (HWE $p$ ), and inbreeding coefficient $\left(F_{I S}\right)$.

\begin{tabular}{lrlllllllll}
\hline \multicolumn{1}{c}{$\begin{array}{c}\text { Sampled } \\
\text { group }{ }^{1,2,3}\end{array}$} & $\mathrm{~N}$ & $\mathrm{~N}_{\mathrm{A}}$ & \multicolumn{1}{c}{$\mathrm{A}_{\mathrm{R}}$} & $\mathrm{N}_{\mathrm{AP}}$ & $\mathrm{A}_{\mathrm{RP}}$ & \multicolumn{1}{c}{$\mathrm{H}_{\mathrm{O}}$} & \multicolumn{1}{c}{$\mathrm{H}_{\mathrm{E}}$} & \multicolumn{1}{c}{$\mathrm{SE}$} & $\mathrm{HWE} p$ & \multicolumn{1}{c}{$\mathrm{F}_{\mathrm{IS}}$} \\
\hline Buff & 13 & 4.80 & 4.64 & 0.000 & 0.02 & 0.569 & 0.582 & 0.058 & 0.0166 & 0.0648 \\
Demi & 46 & 6.47 & 4.97 & 0.067 & 0.06 & 0.654 & 0.658 & 0.041 & 0.4498 & 0.0181 \\
Iris & 25 & 4.87 & 4.34 & 0.133 & 0.09 & 0.519 & 0.581 & 0.060 & $>0.0001$ & 0.1141 \\
Mahogany & 31 & 6.27 & 5.09 & 0.133 & 0.11 & 0.624 & 0.667 & 0.052 & 0.0001 & 0.0872 \\
Pastel & 18 & 4.93 & 5.7 & 0.000 & 0.04 & 0.593 & 0.604 & 0.063 & 0.0284 & 0.0468 \\
NSF & 173 & 7.60 & 4.67 & 0.467 & 0.07 & 0.601 & 0.636 & 0.051 & $>0.0001$ & 0.0546 \\
NBW & 13 & 5.80 & 5.56 & 0.067 & 0.29 & 0.615 & 0.685 & 0.040 & 0.005 & 0.1411 \\
NSW & 84 & 8.67 & 5.79 & 0.400 & 0.13 & 0.614 & 0.717 & 0.043 & $>0.0001$ & 0.1483 \\
PEIW & 85 & 7.47 & 5.11 & 0.067 & 0.13 & 0.629 & 0.704 & 0.036 & $>0.0001$ & 0.1115 \\
Historic & 20 & 6.87 & 5.96 & 0.200 & 0.31 & 0.597 & 0.694 & 0.044 & $>0.0001$ & 0.1695 \\
\hline
\end{tabular}

${ }^{1} \mathrm{NSF}=$ domestic mink from farms in Nova Scotia; NBW = free-ranging mink sampled in New Brunswick; NSW = free-ranging mink sampled in Nova Scotia; PEIW = free-ranging mink sampled in Prince Edward Island

${ }^{2}$ Buff, Demi, Iris, Mahogany, and Pastel mink are different colour lines sampled from farms in Ontario, Canada

${ }^{3}$ Historic mink were Maritime mink from the 1980 s or older and sampled in museums 
Table 4. Mean (SE) membership coefficients of sampled American mink (Neovison vison Schreber, 1777) populations to each group identified in a STRUCTURE analysis when $\mathrm{K}=5$.

\begin{tabular}{|c|c|c|c|c|c|}
\hline Sampled group ${ }^{1,2}$ & Black & Brown & Mahogany & Iris & Wild \\
\hline $\begin{array}{l}\text { Nova Scotia Farm } \\
(n=173)\end{array}$ & $\begin{array}{l}0.585 \\
(0.031)\end{array}$ & $\begin{array}{l}0.040 \\
(0.006)\end{array}$ & $\begin{array}{l}0.345 \\
(0.030)\end{array}$ & $\begin{array}{l}0.020 \\
(0.002)\end{array}$ & $\begin{array}{l}0.011 \\
(0.002)\end{array}$ \\
\hline $\begin{array}{l}\text { Buff } \\
(n=13)\end{array}$ & $\begin{array}{l}0.012 \\
(0.004)\end{array}$ & $\begin{array}{l}0.884 \\
(0.066)\end{array}$ & $\begin{array}{l}0.067 \\
(0.046)\end{array}$ & $\begin{array}{l}0.016 \\
(0.010)\end{array}$ & $\begin{array}{l}0.021 \\
(0.015)\end{array}$ \\
\hline $\begin{array}{l}\text { Demi } \\
(n=46)\end{array}$ & $\begin{array}{l}0.054 \\
(0.018)\end{array}$ & $\begin{array}{l}0.828 \\
(0.035)\end{array}$ & $\begin{array}{l}0.076 \\
(0.024)\end{array}$ & $\begin{array}{l}0.033 \\
(0.008)\end{array}$ & $\begin{array}{l}0.009 \\
(0.002)\end{array}$ \\
\hline $\begin{array}{l}\text { Iris } \\
(n=25)\end{array}$ & $\begin{array}{l}0.015 \\
(0.005)\end{array}$ & $\begin{array}{l}0.022 \\
(0.008)\end{array}$ & $\begin{array}{l}0.013 \\
(0.002)\end{array}$ & $\begin{array}{l}0.940 \\
(0.016)\end{array}$ & $\begin{array}{l}0.010 \\
(0.003)\end{array}$ \\
\hline $\begin{array}{l}\text { Mahogany } \\
(n=31)\end{array}$ & $\begin{array}{l}0.082 \\
(0.023)\end{array}$ & $\begin{array}{l}0.089 \\
(0.038)\end{array}$ & $\begin{array}{l}0.758 \\
(0.056)\end{array}$ & $\begin{array}{l}0.045 \\
(0.019)\end{array}$ & $\begin{array}{l}0.026 \\
(0.006)\end{array}$ \\
\hline $\begin{array}{l}\text { Pastel } \\
(n=18)\end{array}$ & $\begin{array}{l}0.019 \\
(0.005)\end{array}$ & $\begin{array}{l}0.784 \\
(0.060)\end{array}$ & $\begin{array}{l}0.104 \\
(0.045)\end{array}$ & $\begin{array}{l}0.034 \\
(0.023)\end{array}$ & $\begin{array}{l}0.058 \\
(0.026)\end{array}$ \\
\hline $\begin{array}{l}\text { NS, NB, and PEI free- } \\
\text { ranging } \\
(n=182)\end{array}$ & $\begin{array}{l}0.214 \\
(0.027)\end{array}$ & $\begin{array}{l}0.056 \\
(0.011)\end{array}$ & $\begin{array}{l}0.059 \\
(0.011)\end{array}$ & $\begin{array}{l}0.013 \\
(0.002)\end{array}$ & $\begin{array}{l}0.658 \\
(0.031)\end{array}$ \\
\hline $\begin{array}{l}\text { Historic } \\
(n=20)\end{array}$ & $\begin{array}{l}0.036 \\
(0.014)\end{array}$ & $\begin{array}{l}0.072 \\
(0.032)\end{array}$ & $\begin{array}{l}0.026 \\
(0.008)\end{array}$ & $\begin{array}{l}0.016 \\
(0.006)\end{array}$ & $\begin{array}{l}0.851 \\
(0.048)\end{array}$ \\
\hline
\end{tabular}

${ }^{1}$ Buff, Demi, Iris, Mahogany, and Pastel mink are different colour lines sampled from farms in Ontario, Canada

${ }^{2}$ Historic mink were Maritime mink from the 1980 s or older and sampled in museums 
Table 5. Summary statistics for genetic groups of American mink (Neovison vison Schreber, 1777 ) identified in Structure when $K=5$. Data include historic samples but all hybrid individuals $(0.2<q>0.8 ; N=96)$ were removed. Number of individuals in each group $(N)$ are shown, along with number of alleles $\left(N_{A}\right)$, allelic richness $\left(A_{R}\right)$, number of private alleles $\left(N_{A P}\right)$, private allelic richness $\left(A_{R P}\right)$, observed heterozygosity $\left(H_{O}\right)$, expected heterozygosity $\left(H_{E}\right)$, standard error $(S E)$ of $H_{E}$, and probability of deviations from Hardy-Weinberg Equilibrium (HWE $p$ ), and inbreeding coefficient $\left(F_{I S}\right)$.

\begin{tabular}{lrlllllllll} 
Population & $\mathrm{N}$ & $\mathrm{N}_{\mathrm{A}}$ & $\mathrm{A}_{\mathrm{R}}$ & $\mathrm{N}_{\mathrm{AP}}$ & $\mathrm{A}_{\mathrm{RP}}$ & $\mathrm{H}_{\mathrm{O}}$ & $\mathrm{H}_{\mathrm{E}}$ & $\mathrm{SE}$ & $\mathrm{HWE} p$ & $\mathrm{~F}_{\text {IS }}$ \\
\hline Black & 129 & 7.400 & 5.48 & 0.400 & 0.15 & 0.604 & 0.638 & 0.051 & 0.0000 & 0.0540 \\
Brown & 60 & 6.800 & 5.88 & 0.133 & 0.23 & 0.607 & 0.660 & 0.049 & 0.0001 & 0.0885 \\
Mahogany & 70 & 8.467 & 6.97 & 0.267 & 0.32 & 0.587 & 0.719 & 0.051 & 0.0000 & 0.1908 \\
Iris & 24 & 5.933 & 5.84 & 0.000 & 0.15 & 0.593 & 0.652 & 0.040 & 0.0000 & 0.1125 \\
Wild & 129 & 8.667 & 6.64 & 0.533 & 0.56 & 0.619 & 0.726 & 0.037 & 0.0000 & 0.1491 \\
\hline
\end{tabular}


Table 6. Pairwise $F_{S T}$ values for all groups of American mink (Neovison vison Schreber, 1777) identified through Structure analysis. Hybrid individuals were removed for calculations. F $_{\text {ST }}$ values are below the diagonal and probabilities (based on 9999 permutations) are above the diagonal.

\begin{tabular}{rrrrrl}
\hline \multicolumn{1}{l}{ Black } & Brown & Mahogany & \multicolumn{2}{l}{ Iris } & \multicolumn{2}{l}{ Wild } \\
\hline-- & 0.000 & 0.000 & 0.000 & 0.000 & Black \\
0.050 & -- & 0.000 & 0.000 & 0.000 & Brown \\
0.049 & 0.043 & -- & 0.000 & 0.000 & Mahogany \\
0.022 & 0.068 & 0.064 & -- & 0.000 & Iris \\
0.089 & 0.099 & 0.056 & 0.078 & -- & Wild \\
\hline
\end{tabular}




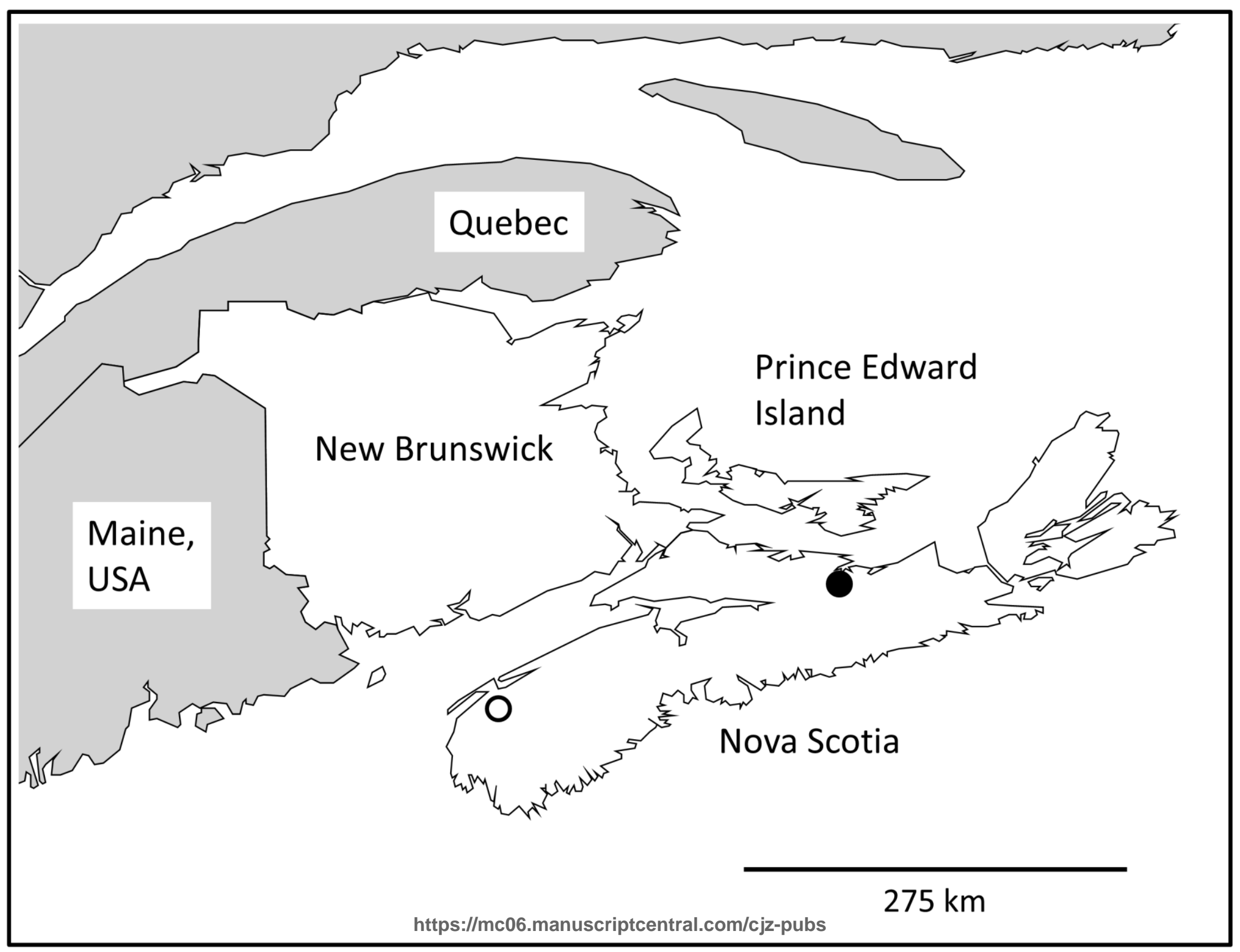


(a)

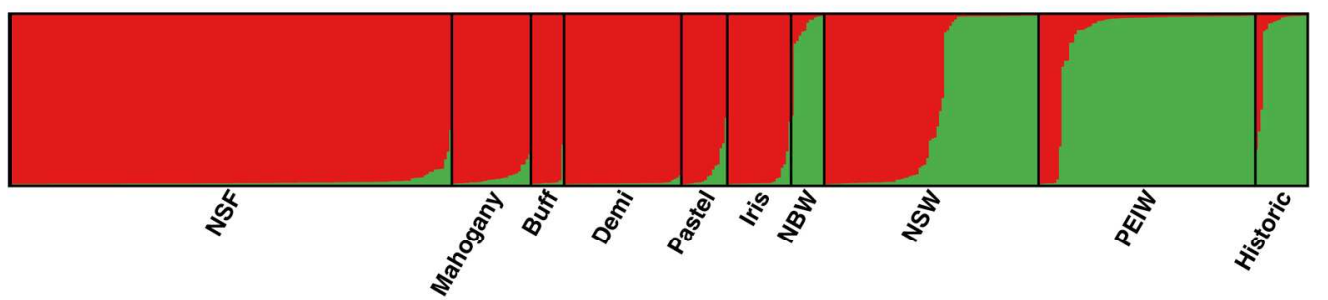

(b)

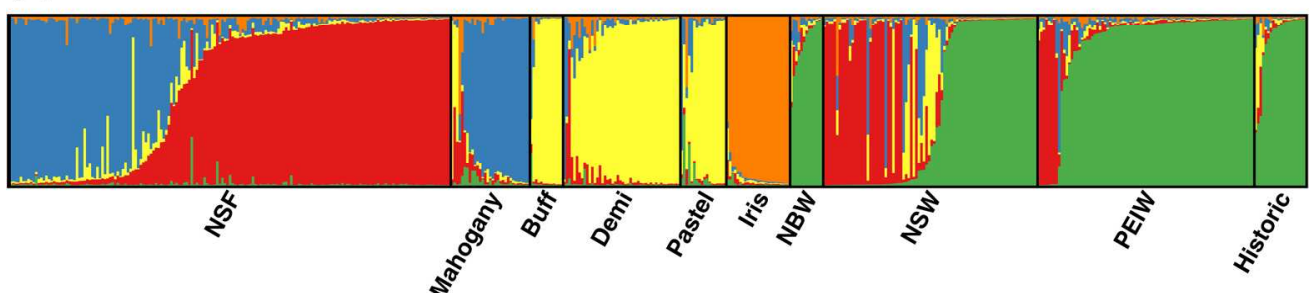

Caption : Figure 2. Bar plots for population structure analysis and identification of admixed American mink (Neovison vison) individuals using Structure v.2.3.3 (Pritchard et al. 2000) at $K=2$ (a) and $K=5$ (b). Individuals are grouped by known sampling locations and/or colour phase ( $\mathrm{X}$ axis) separated by black vertical lines. Each coloured vertical bar represents one individual and different colours show group membership coefficients $(q) . K=2$ discriminated domestic mink (red) from wild minks (green) whereas $\mathrm{K}=$ 5 identified wild mink (green) as a separate group and additionally differentiated according to fur colour within domestic farm mink (black mink = red; brown mink = yellow; iris mink = orange; mahogany mink = blue). NBW = New Brunswick free-ranging mink, NSW = Nova Scotia free-ranging mink, PEI = Prince Edward Island free-ranging mink, and NSF = Nova Scotia domestic mink.

Fig. 2

$180 \times 95 \mathrm{~mm}(300 \times 300 \mathrm{DPI})$ 


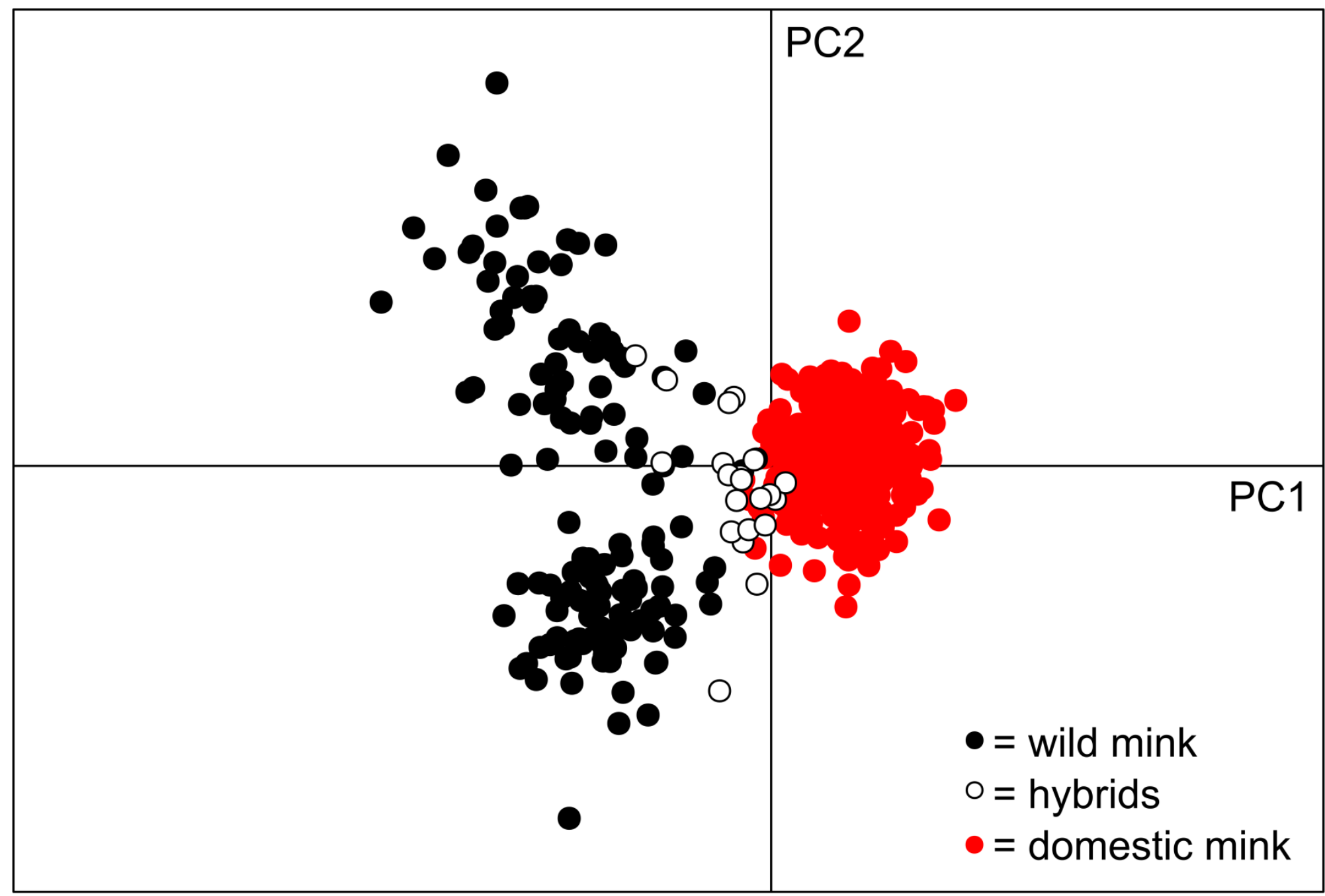

https://mc06.manuscriptcentral.com/cjz-pubs 


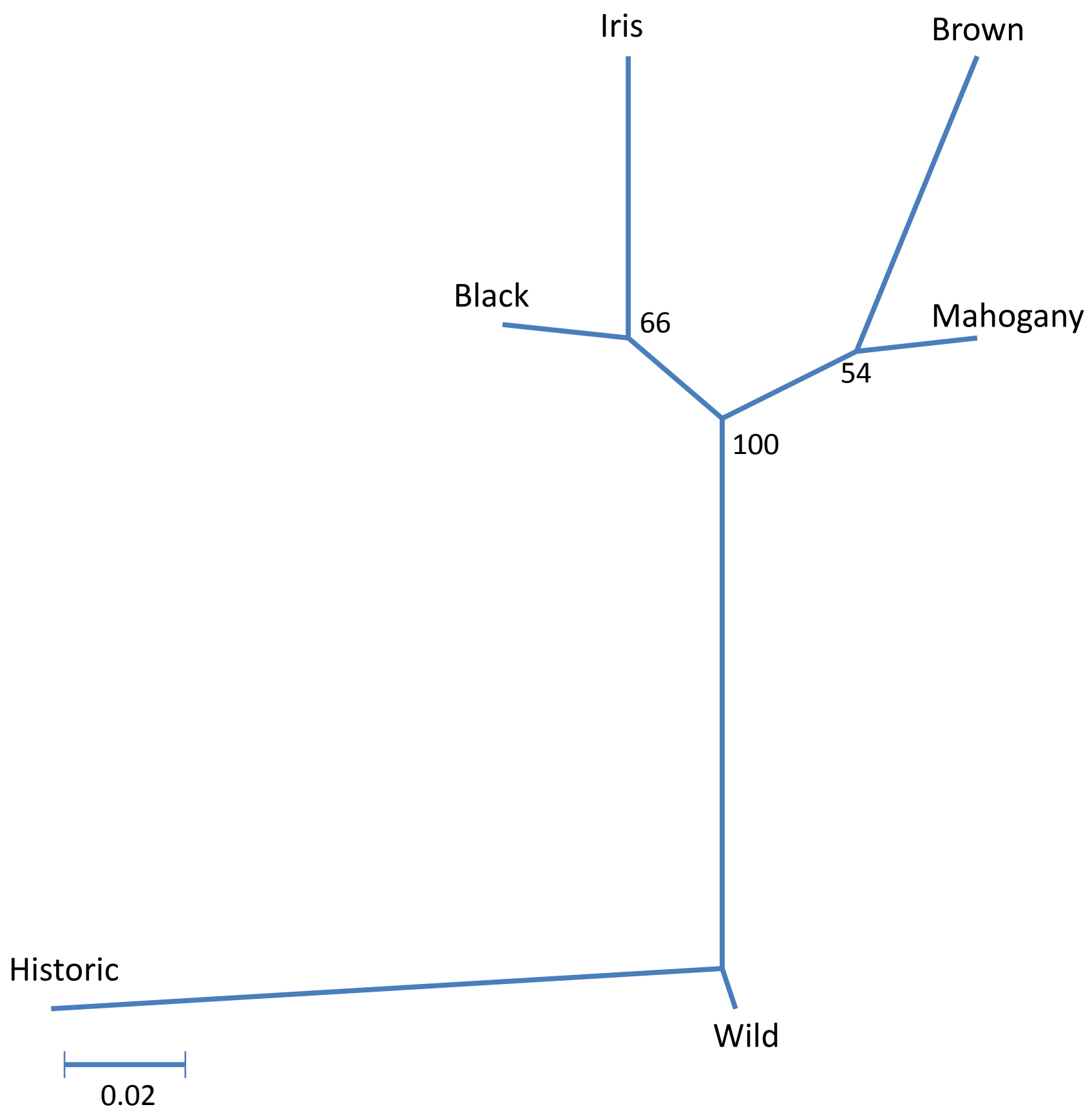

